\section{Spatial summation and the dynamics of warmth sensation*}

\author{
JOSEPH C. STEVENS $†$ and LAWRENCE E. MARKS \\ John B. Pierce Foundation Laboratory and Yale University \\ New Haven, Connecticut 06519
}

Various areal extents of the forehead and back were thermally irradiated at various levels of intensity. For any areal extent, the degree of apparent warmith grows approximately as a power function of intensity level; the larger the area, the smaller the exponent of the power function. Two families of psychophysical functions, one for the forehead, the other for the back, both show that the power functions extrapolate to a point of convergence in the neighborhood of the threshold for pain and tissue impairment. The rules that govern spatial summation of warmth reveal themselves in the two families. Intensity and area trade one for the other to preserve the same level of warmth. At faint sensation levels, reciprocity is the rule of trading; but with increasing sensation level, area makes a weaker and weaker relative contribution to warmth, and, as a result, it takes a larger and larger percentage change in area to offset a given percentage change in intensity.

Several sense modalities exhibit spatial summation, perhaps none so impressively as the sense of warmth. Summation takes place generously over large portions of the body surface. In the first extensive quantitative measurements of warmth summation, Hardy and Oppel (1937) found: (1) almost complete summation over the forehead and over the back of the hand and forearm (i.e., radiant intensity and areal extent could be traded one for the other to preserve the threshold of warmth); (2) "partial" summation between the two hands (i.e., the threshold level for one hand was about $50 \%$ greater than for two hands); and (3) no evidence of summation between hand and forehead. Later Kenshalo, Decker, and Hamilton (1967) found virtually complete summation over portions of the forehead, the forearm, and the back; this was true whether the skin was warmed by irradiation or by conduction.

The first purpose of the present experiment was to learn whether the same rules that govern summation at threshold apply throughout the functional range of the warmth sense. The behavior at threshold sometimes does, sometinies does not, prove a reliable index of the supralininal sensitivity of a sense modality (see Marks, 1968). That spatial summation does take place above the threshold was demonstrated by Herget, Granath, and Hardy (1941), but the data do not tell whether summation might vary

*This rescarch was supported in part under USPHS Grant ES-00354-(1) and in part under Contraet F44620-67-C-0017 with the Air Foree Office of Scientific Research.

tAddress: John B. Picrce Foundation Laboratory, 290 Congress Avenue, New Haven, Connecticut 06519.

Two regions of the body werc systematically with sensation level. It is known, however, that pain inflicted by radiation exhibits little or no spatial summation (Hardy, Wolff, \& Goodell, 1940; Greene \& Hardy, 1958; Murgatroyd, 1964)

A second purpose, closely related to the first, was to learn whether the growth rate of apparent warmith with thermal intensity depends on how much of the skin is stimulated. Earlicr scaling (J.C. Stevens \& Marks, 1967; Marks \& Stevens, 1968b) established that warmth aroused by brief irradiation of the front and back of the body conforms to a general psychophysical power law (S. S. Stevens, 1961):

$$
\psi=\mathrm{k}\left(\varphi-\varphi_{\mathrm{o}}\right)^{\beta}
$$

where $\psi$ stands for apparent magnitude, $\varphi$ for plyysical magnitude, and $\varphi_{0}$ for the minimally perceptible physical magnitude. The question here is whether the constants $\beta$ and $k$ in Eq. 1 depend on areal extent (since $\varphi_{0}$ approximates threshold, its size must decrease in near proportion to area). examined-the forehead because it has highly uniform surface temperature and absolute sensitivity, and the back because

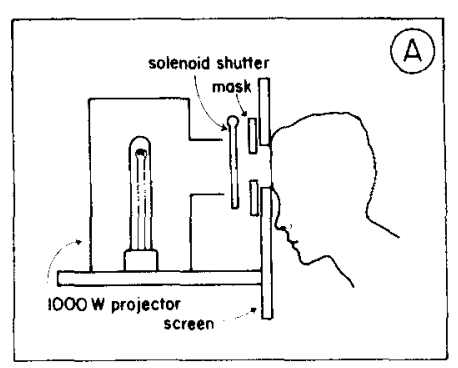

Fig. 1. (a) The setup for Experiment 1; (b) the setup for Experiment 2. it forms a conveniently large and relatively flat surface. As it turned out, the rules of summation and the behavior of the constants of the psychophysical power functions are fairly similar for these two regions, even though in terms of unit area the forehead is much more sensitive than the back (Hardy \& Oppel, 1937; Kenshalo, Decker, \& Hamilton, 1967).

\section{APPARATUS}

The experiments took place in an air-conditioned room at $21^{\circ} \mathrm{C}$ and at low relative humidity.

\section{Experiment 1 (Forehead)}

A 1,000-W projector lamp was positioned to cast uniform irradiation on the forehead (Fig. 1a). Intensity level was controlled by varying the voltage to the lamp. To promote absorption of irradiation throughout the spectrum emitted by the lamp, the forehead was painted with India ink. Radiant intensity was measured with a Hardy radiometer (Hardy, Wolff, \& Goodell, 1952) and ranged from 18.8 to $209 \mathrm{~mW} / \mathrm{cm}^{2}$ (4.5 to $\left.50 \mathrm{mcal} / \mathrm{sec} \times \mathrm{cm}^{2}\right)$.

The area extent of irradiation (2.55 to $21.7 \mathrm{~cm}^{2}$ ) was determined by aluminum masks (ratio of width to height: $1.5: 1.0$ ) placed directly in front of the forehead. A thin metallic screen, lined with cork on the S's side, shielded the rest of the body from the radiation and blocked sight of the projector lamp. The duration of exposures was fixed at $3 \mathrm{sec}$ with a timed shutter.

Size and slant of the forehead varies from person to person. For the sake of comfort, the $S$ sat in a chair that permitted wide adjustment of height, tilt, and head position.

\section{Experiment 2 (Back)}

As is sketched in Fig. Ib, the $S$ sat in a chair having in its wooden back a large aperture exposed to four heat lamps. Each lamp was a General Electric T-3 quartz lamp, $40 \mathrm{~cm}$ long, mounted in a parabolic-cylindrical reflector made of stainless steel. The lamps were spaced to cast uniform irradiation on the back.

Intensity level was controlled by varying the voltage to the lamps. For the reason

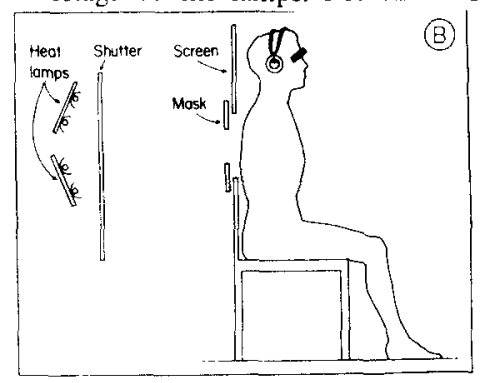




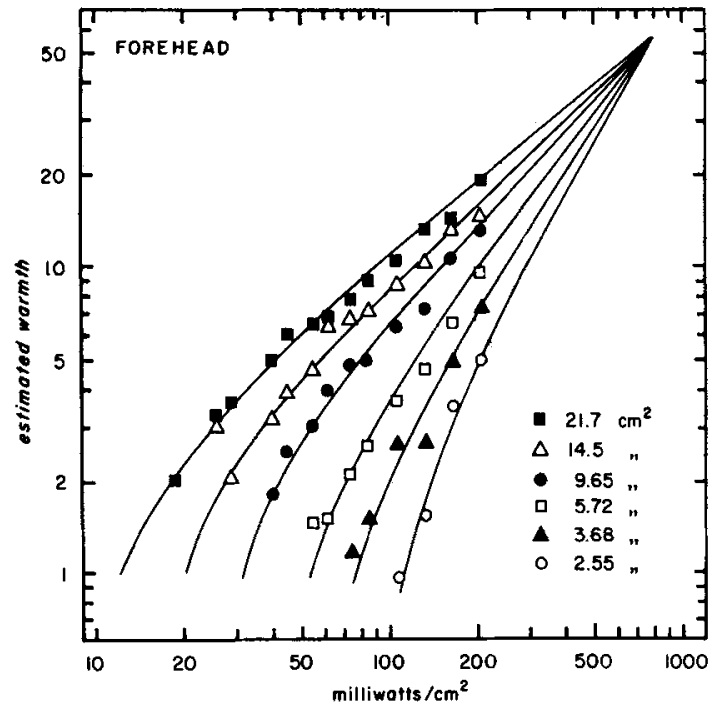

Fig. 2. Magnitude estimation of warmth as a function of radiant intensity applied to the forehead. The parameter is areal extent of the stimulation.

stated above, the S's back was painted with India ink. Flux level ranged from 36 to $282 \mathrm{~mW} / \mathrm{cm}^{2}$ (8.6 to $67.5 \mathrm{mcal} / \mathrm{sec} X$ $\mathrm{cm}^{2}$ ).

Areal extent was determined by aluminum masks, each having a circular aperture whose center lay $42 \mathrm{~cm}$ above the seat of the chair. Area ranged from 31.4 to $324 \mathrm{~cm}^{2}$. The $S$ was blindfolded to prevent visual cues. Duration was fixed at approximately $3 \mathrm{sec}$ per exposure by means of a hand-operated aluminum shutter.

\section{PROCEDURE}

\section{Experiment 1 (Forehead)}

Each of 18 males served in two practically identical sessions, separated by a day or longer. Their task was magnitude estimation of warmth, i.e., the assignment of numbers to match the degree of warmth experienced on exposures to the radiation. An exposure occurred once every $30 \mathrm{sec}$.

Each S chose his own "modulus," i.e., he selected any number he deemed appropriate to stand for the degree of warmth experienced on the first exposure. Intensity level and area of this first exposure were deliberately varied from $S$ to $S$, but were always selected so as to arouse moderate warmth. For practice the $\mathrm{S}$ was then given four more exposures to other intensity-area combinations. Finally, 60 different combinations of level and area were presented and estimated. The order of presentation was random with respect to perceived level of warmth, except that it was deemed advisable not to present a very low-level exposure on the next trial after the highest level.

As judged from the responses of the 18 Ss, sensitivity to warmth varied to some extent from person to person. The data from three Ss were not counted because they felt nothing on a large proportion of the trials. From the remaining Ss, seven of the intensity-area combinations drew numerous estimations of zero. The data on these combinations were, therefore, not counted.

\section{Experiment 2 (Back)}

The procedures resembled closely those of Experiment 1, except for the following differences: (1) The stimulus combinations were selected so as to avoid estimates of zero warmth (in contrast to Experiment 1, no zero estimates were given). (2) There were fewer stimulus combinations-36 compared to 60. (3) A stimulation was given once rather than twice every minute. (4) Fourteen Ss were tested, only one of whom had served in Experiment 1.

\section{RESULTS}

Psychophysical Functions for the Forehead

For each intensity-area combination, the 30 estimates ( 2 from each of $15 \mathrm{Ss}$ ) were averaged geometrically; the geometric means are plotted in the $\log$-log coordinates of Fig. 2 as a function of radiant intensity in milliwatts/square centimeter. The points determine a family of psychophysical functions, one for each stimulus area. The main feature of this family is that subjective warmth grows regularly stronger with radiant intensity, but the rate of growth depends on area: the smaller the area, the faster the growth rate. On extrapolation, the functions tend to converge on a point near $800 \mathrm{~mW} / \mathrm{cm}^{2}$; this level lies in the neighborhood of the threshold for pricking pain (see Adair, Stevens, \& Marks, 1968, for a review of various measurements of the pain threshold). The curves in Fig. 2 have the form of Eq. 1, which was earlier found to describe the relationship between warmth and radiant intensity (J.C. Stevens \& Marks, 1967; Marks \& Stevens, 1968b). The constants $\varphi_{0}, k$, and $\beta$ all depend on area. The next section describes how the values of these constants were estimated.

\section{Estimation of the Constants of the Warmth Functions}

A realistic estimate of $\varphi_{0}$ can sometimes be made with an iterative least-squares procedure. (For examples, see J. C. Stevens \& Marks, 1967; Adair, Stevens, \& Marks, 1966; and Marks \& Stevens, 1968b.) Briefly stated, for each of several conceivable sizes of $\varphi_{0}$, a power function of the form of Eq. 1 is fitted to the data by the method of least squares. For each function so fitted, the Pearson $r$ is computed to show the strength of the relation between the Ss' magnitude estimations (log geometric means) and the logs of $\varphi-\varphi_{0}$. The value of $\varphi_{0}$ that maximizes $r$ is taken as the estimate of the effective threshold,

Unfortunately, this statistical procedure yielded a realistic answer for only three of the six psychophysical functions. For the other three functions the "best" value of $\varphi_{0}$ turned out to be zero or negative, meaning that an effective threshold cannot be inferred from the data points themselves. This is not surprising, given a degree of "noisiness" obvious in Fig. 2. Because a good estimate of the exact sizes of $\beta$ and $\mathrm{k}$ might depend on the estimate of the size of $\varphi_{0}$, the iterative least-squares solutions were supplemented by least-squares solutions made under the assumption that $\varphi_{0}$ equals zero. Fortunately, the differences in the answers given by the two methods are not of overriding importance.

This is demonstrated in Fig. 3, where the size of $\mathrm{k}$ obtained by the two least-squares methods is plotted as a function of the size of $\beta$ obtained. The relationship between $\log \mathrm{k}$ and $\beta$ approximates linearity. $A$ linear relationship between $\log k$ and $\beta$ is a necessary property of a family of power functions that intersect at a common point (J. C. Stevens \& Rubin, 1970). Consider a family of functions of the form $\log \psi^{\prime}=$ $\beta \log \varphi+\log k$ and assume they intersect at a point $\log \psi=\mathrm{a}, \log \varphi=\mathrm{c}$. Then

$$
a=c \beta+\log k \text { or } \log k=a-c \beta
$$

The slope $c$ of the line in Fig. 3 relates to the point of intersection of the power functions. The slope of the line drawn inuplies that the functions converge at a radiant intensity of about $800 \mathrm{~mW} / \mathrm{cm}^{2}$, or 


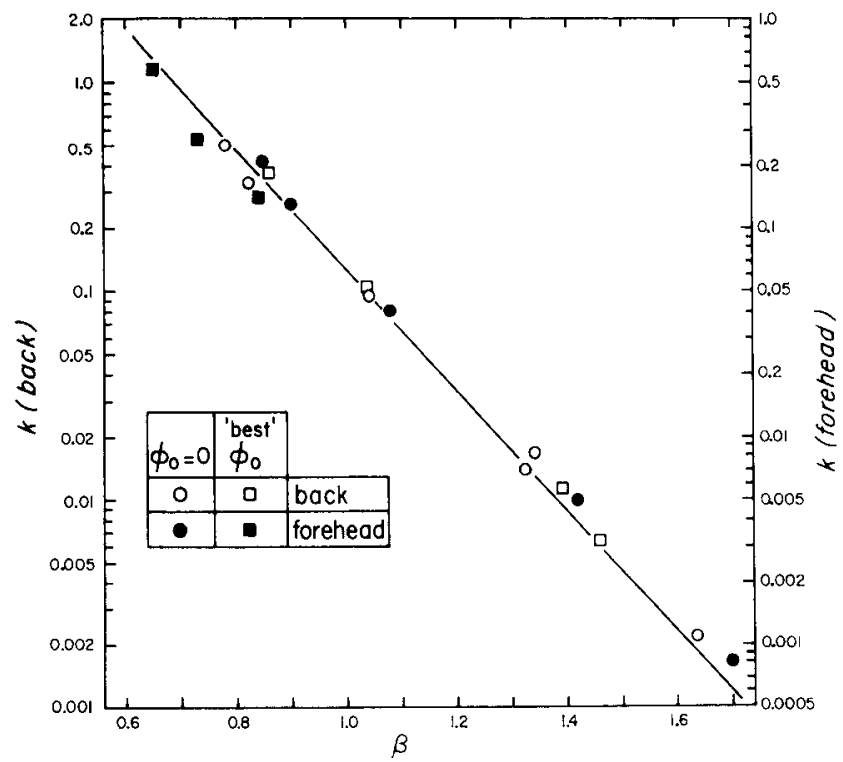

Fig. 3. How $\mathrm{k}$ varies as a function of $\beta$ for the various areal extents of stimulation. The values of $k$ and $\beta$ were calculated with (squares) and without (circles) a threshold correction, $\varphi_{o}$. See text.

approximately the threshold of pricking pain and tissue impairment. In fact, the line in Fig. 3 is slightly too steep to constitute the best fit to the data; the convergence point may, therefore, lie slightly below the actual pain threshold. The point here is that warmth sensation probably ceases to depend on area in the near vicinity of, if not precisely at, the pain threshold.

The curve-fitting procedures demonstrate what is rather obvious from straightforward inspection of Fig. 2, namely, that degree of warmth depends progressively more on radiant intensity and progressively less on area as warmth approaches a noxious level. Only at low sensation levels do area and intensity make approximately equal contributions to the sensory effect.

\section{"Idealized" Family of Warmth Functions}

As a last step in the assessment of $\beta$ and $k$, power functions were fitted to the geometric means by least-squares solutions, done this time under the restriction that the fitted functions pass through a common point at $800 \mathrm{~mW} / \mathrm{cm}^{2}$. The estimates of $\varphi_{0}$ were based in part on casual threshold measurements on five of the Ss, and in part on results of the iterative least-squares procedure described above. The values of $\varphi_{0}$ were selected to be consistent both with these measurements and with the assumption (supported by the measurements of Hardy \& Oppel, 1937. and Kenshalo, Decker, \& Hamilton, 1967) that to a first approximation $\varphi_{0}$ is related to Area $A$ by the formula $\varphi_{0} A=b$. The constants $\beta, \mathrm{k}$, and $\varphi_{0}$ were used 10 $800 \mathrm{~mW} / \mathrm{cm}^{2}$.
Accordingly, no attempt was made in Fig. 5 to depict the "downturn" in the psychophysical function that characteristically occurs near threshold (Marks \& Stevens, 1968a).

The relationship of $\mathbf{k}$ to $\beta$ (determined by two kinds of least-squares solutions, as described above) closely resembles that for the forehead (see Fig. 3), except that the back yielded sizes of $\mathrm{k}$ approximately twice those of the forehead. (The absolute size of $\mathrm{k}$ cannot be compared meaningfully from experiment to experiment because the Ss were free to adopt any modulus.) That the same slope fits both sets of data in Fig. 3 demonstrates that both families of power functions (Fig. 2 and Fig. 5) converge at approximately the same level of radiant intensity.

As a last step in the assessment of $\beta$ and $k$, power functions were fitted to the geometric means by least squares, under the restriction that the fitted functions pass through a common point at $800 \mathrm{~mW} / \mathrm{cm}^{2}$. The constants thereby obtained were used to construct the curves in Fig. 5. These same constants are also plotted in Fig. 4 as a function of area. The basic relationships of $\beta$ and $k$ to area look much the same for back and forehead-except, of course, that the areas of the back are about 20 times those of the forehead. For example, Fig. 4 suggests that irradiation of about $7 \mathrm{~cm}^{2}$ of the forehead and about $160 \mathrm{~cm}^{2}$ of the back would both yield an exponent equal to 1.0. This finding reflects the fact that, in terms of unit area, the forehead is much more sensitive to warmth than is the back (Hardy \& Oppel, 1937; Kenshalo, Decker, \& Hamilton, 1967).

\section{Equal-Warmth Contours}

The families of power functions in Figs.

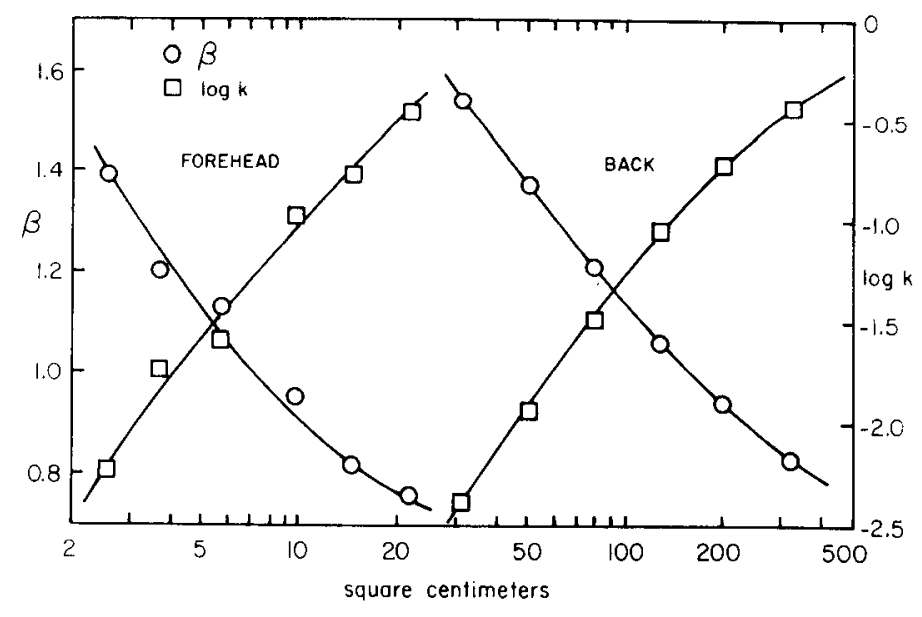

Fig. 4. How $\beta$ (circles) and log $k$ (squares) vary as functions of area under the assumption that all of the psychophysical functions pass through a common point at 


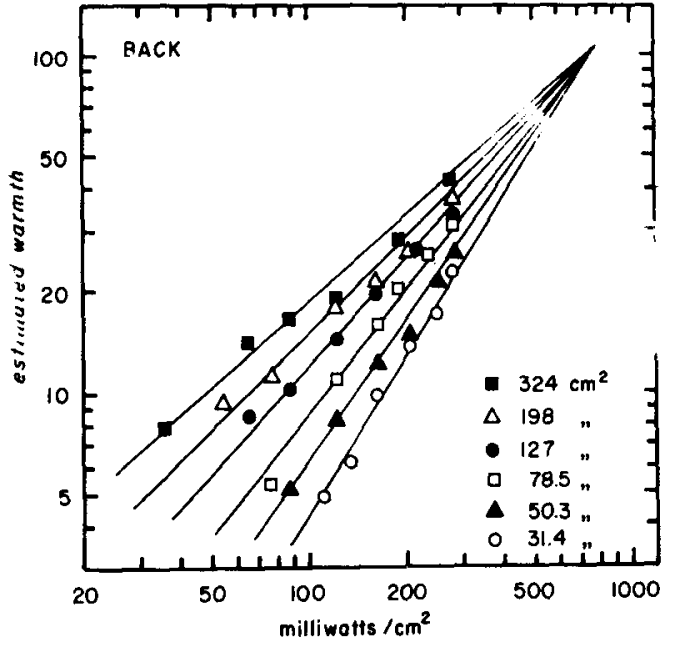

Fig. 5. Magnitude estimation of warmth as a function of radiant intensity applied to the back. The parameter is areal extent of stimulation.

2 and 5 contain the information needed to specify the combinations of radiant intensity and area that arouse the same degree of warmth. Figures 6 and 7 show representative equal-warmth contours for forehead and back. These were generated by making horizontal cuts at various levels of $\psi$ in Figs. 2 and 5. The points in Figs. 6

and 7 represent the intersections of the cuts with the psychophysical functions.

The equal-warmth contours depict the broad features of spatial summation over the dynamic range of the warmth sense. Near threshold the contours have maximal steepness, indicating rich summation. A slope of -1.0 in $\log \cdot \log$ coordinates (shown in Fig. 6 by the line labeled "threshold" means that area and radiant intensity ca be traded equally one for the oth according to the equation $A \varphi=b$ (or $\log$ $=\log \mathrm{b}-\log \mathrm{A})$.

With increasing warmth, however, th contours tend progressively to lo steepness. This means that, as sensatic level increases, area more and more loses i power to contribute to the sensation ( warmth; consequently, the higher th sensation level, the larger percentas change in area it takes to offset a give percentage change in radiant intensity Near a level high enough to cause prickin pain, negligible summation takes plac (Hardy, Wolff, \& Goodell, 1940; Greene ، Hardy, 1958; Murgatroyd, 1964). Th approximate pain level is shown in Figs. and 7 by horizontal dashed lines.

Another feature of Figs. 6 and 7 is th slight curvature of many of the contours $i$ $\log -\log$ coordinates; the curvature suggesi that, with increasing sensation leve summation within relatively small area may fall off proportionally more rapidl than that within larger areas; but this mus remain an unsure, if interesting, poin because of the degree of experiment: uncertainty in the data from which th equal-warmth contours are derived. Not also that some of the contours ar

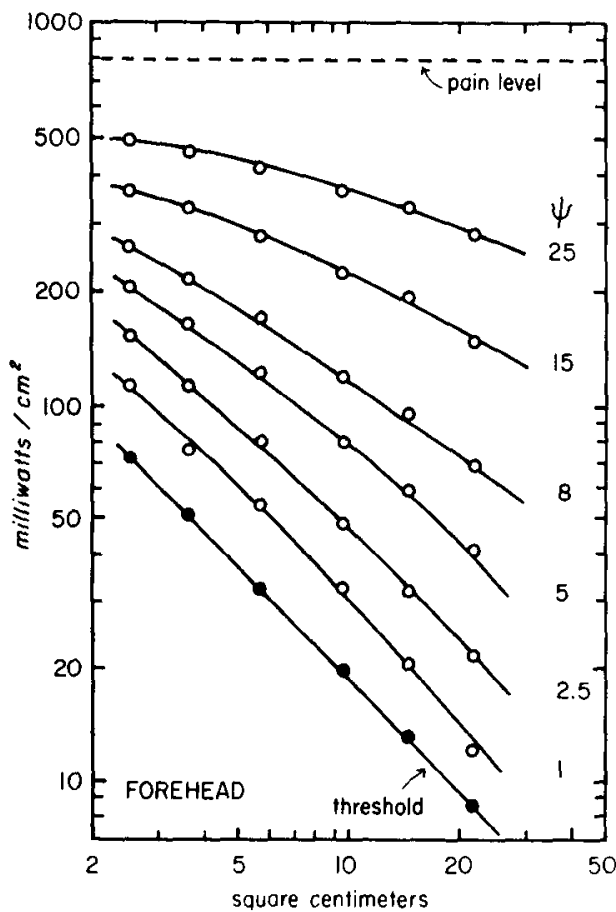

Fig. 6. Equal-sensation contours slowing spatial summation on the forehead at various levels of warmth. The plotted points were determined from horizontal cuts, made at various levels of $\psi$, through the psychophysical functions of Fig. 2. The dashed horizontal line shows absence of spatial summation in the region of the pain threshold. The line labeled "threshold" has a slope of -1.0 .

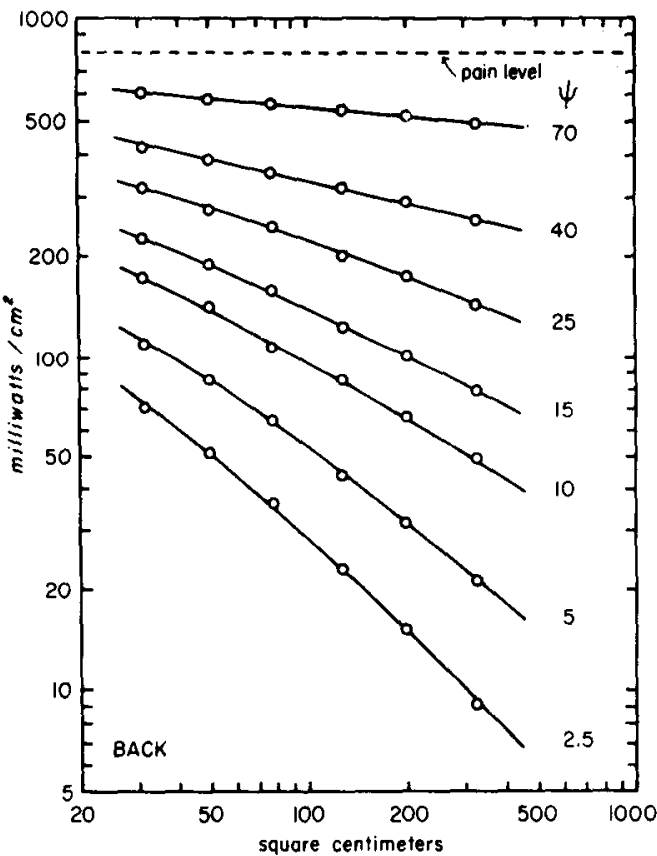

Fig. 7. Equal-sensation contours showing spatial summation on the back at various levels of warmth. The plotted points were determined from horizontal cuts, made at various levels of $\psi$, through the psychophysical functions of Fig. 5. The dashed horizontal line shows absence of spatial summation in the region of the pain threshold. 


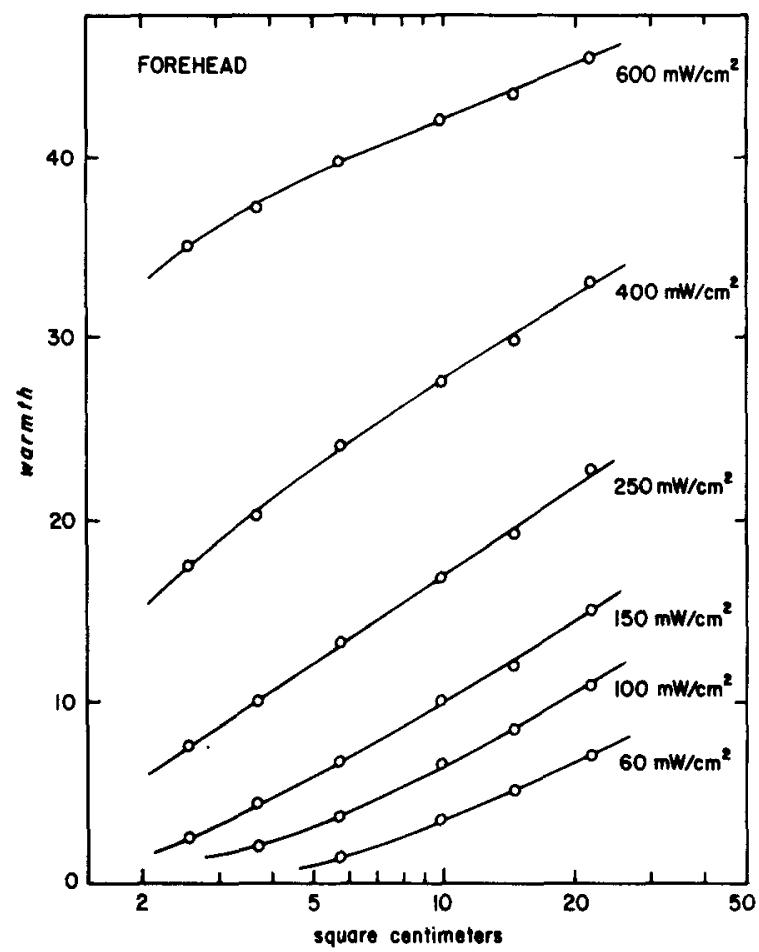

Fig. 8. How warmth grows as a function of the areal extent of stimulation of the forehead. The functions were constructed by making vertical cuts at various levels of irradiance through the psychophysical functions of Fig. 2.

generated from extrapolated portions of the psychophysical functions of Figs. 2 and 5 .

Figures 6 and 7 should not be interpreted as implying that summation must continue to take place for indefinitely small and large areas of stimulation. The nature of the areal limits on summation is discussed by Hardy and Oppel (1937). In practice, the accurate study of tiny areas is limited by the spread of heat laterally over the skin. In theory, the rules of summation must change when the area of the stimulation begins to match the fineness of the receptor "grain." The failure of summation when area becomes large enough was attributed by Hardy and Oppel to the excitation threshold of the most sensitive individual receptor elements; they placed this threshold in the vicinity of $1 \mathrm{~mW} / \mathrm{cm}^{2}$. A stightly lower level, even when cast on the entire body surface, fails to elicit a perceptual reaction, presumably because it cannot trigger the primary receptor elements.

This theory is supported by Hardy and Oppel's observation that just-detectable stimulation of the whole body is experienced as feeling much warmer than just-detectable stimulation of a small area. In other words, although the area of summation is limited by the excitation threshold, once the excitation threshold is cxceeded, the area of summation may expand. It follows that threshold through the psychophysical functions of Fig. 5. measurement may, taken by itself, suggest a misleadingly low areal limitation on summation. The higher the level of subjective warmth, the larger the theoretical areal limit. Figure 6, however, shows that, relative to intensity, area makes a progressively less effective contribution to the experienced warmth as sensation level is increased. Nevertheless, the areal limit on "partial" summation is not yet known, if indeed one exists.

\section{Warmth as a Function of Area}

Apparent warmth grows with area when radiant intensity is maintained constant. This is shown in the semilog coordinates of Fig. 8 (forehead) and Fig. 9 (back). The plotted points represent the intersections of vertical cuts of the psychophysical functions of Figs. 2 and 5. At intermediate levels of radiant intensity, warmth grows approximately as the logarithm of area. At low levels of irradiation, and perhaps at high levels, a simple log function fails.

Figures 8 and 9 are in agreement with a study by Herget, Granath, and Hardy (1941), who asked six Ss to estimate the warmth caused by constant radiation of various extents of the forehead. Two functions were generated (quite similar to each other in shape), one at constant

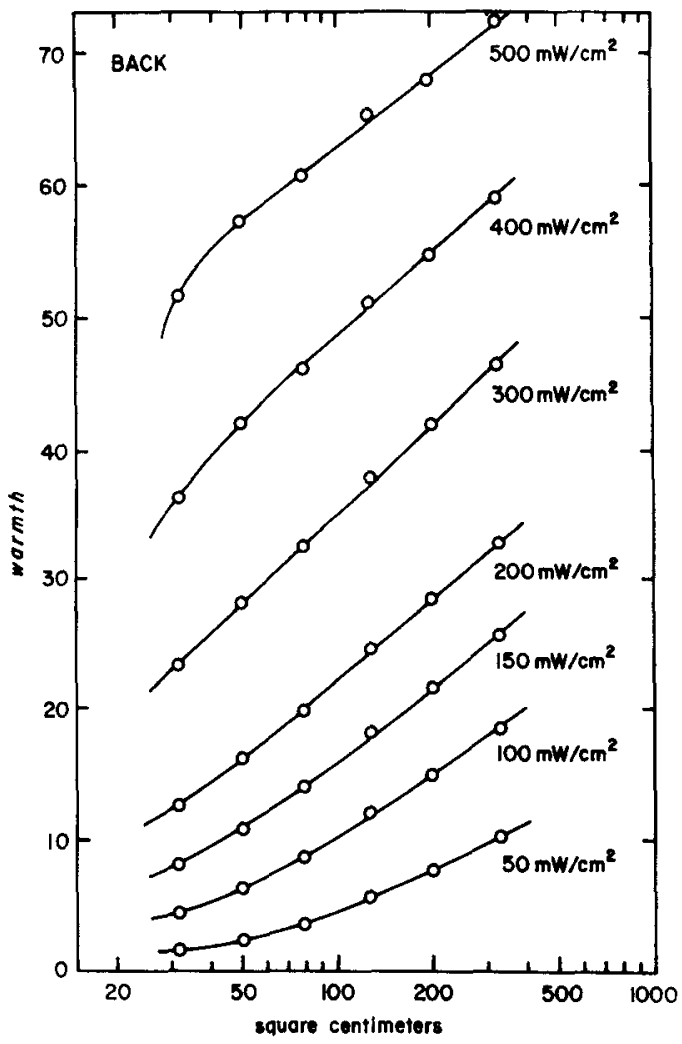

Fig. 9. How warmth grows as a function of the areal extent of stimulation of the back. The functions were constructed by making vertical cuts at various levels of irradiance 


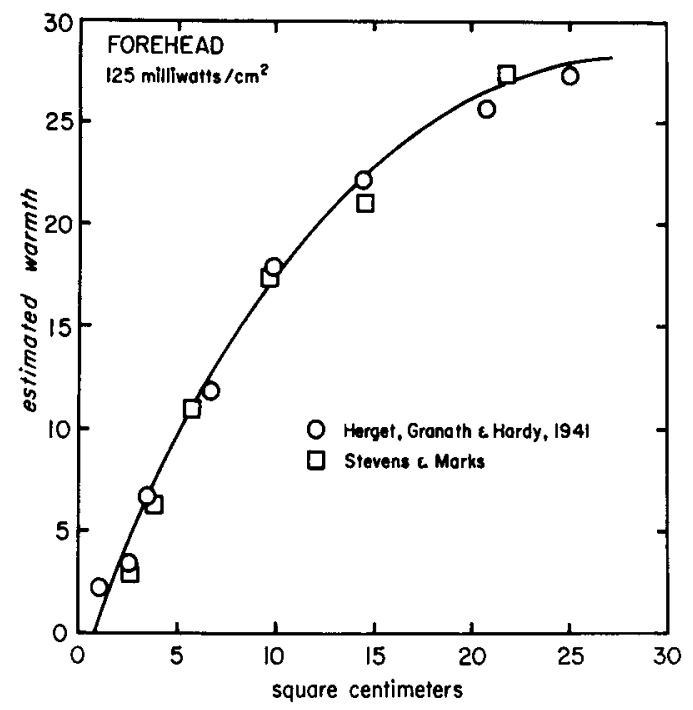

Fig. 10. How warmth varies as a function of the areal extent of stimulation $\left(125 \mathrm{~mW} / \mathrm{cm}^{2}\right)$ of the forehead. The squares are results from the present experiment; the circles are results from Herget, Granath, \& Hardy, 1941.

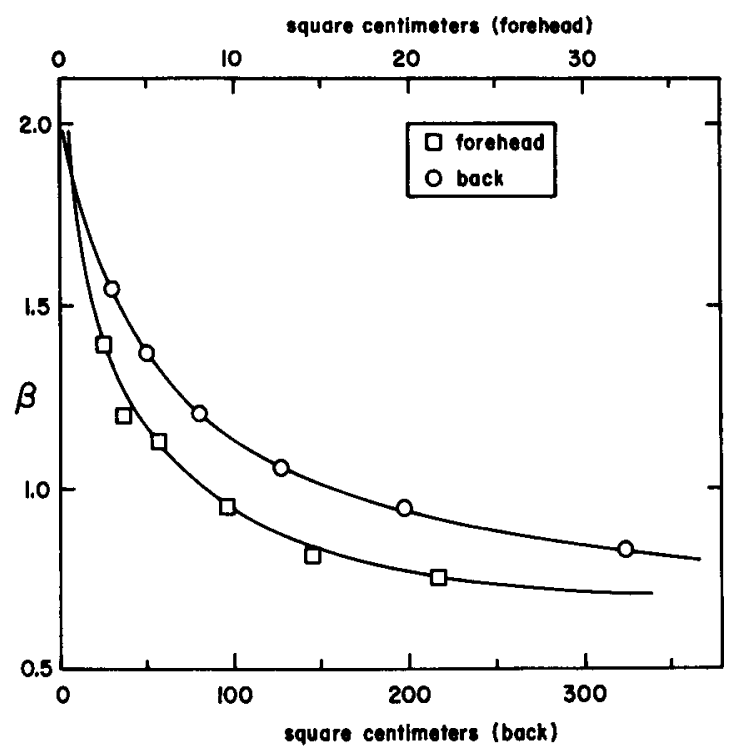

Fig. 11. How $\beta$ varies with the area of stimulation of forehead and back.
$42 \mathrm{~mW} / \mathrm{cm}^{2}$, the other at constant $125 \mathrm{~mW} / \mathrm{cm}^{2}$. For comparison with the present study, Fig. 10 presents the results (circles) at $125 \mathrm{~mW} / \mathrm{cm}^{2}$, together with a vertical cross-section of Fig. 2 at $125 \mathrm{~mW} / \mathrm{cm}^{2}$ (squares). In order to make the results from the two experiments commensurate (because of a modulus difference), it was necessary approximately to double the ordinate values for the squares. Both sets of data are plainly negatively accelerated when plotted in arithmetic coordinates.

\section{Reports on Quality and Extent}

To the degree that the warmih sense displays rich spatial summation, it also displays inferior capacity for spatial discrimination and localization. At low levels of subjective warmth it is virtually impossible to appreciate the spatial aspects of the stimulus. For example, a low-level stimulus applied to a large area and a higher level stimulus applied to a small area may not feel discriminably different. However, the decline in summation with increasing warmth could be accompanied by improvement in spatial acuity. This possibility was examined in an exploratory experiment.

Ten Ss made magnitude estimations of the apparent areal extent of stimulation of the back, under the instruction to ignore the degree of warmth involved. There were nine area-intensity combinations, as given in Table 1. These were chosen with reference to Fig. 5 at three levels of warmth, namely, $\psi=8,12$, and 20 . The cells of Table 1 contain the geometric means of the magnitude estimations. The data were variable, but there is evidence for some degree of spatial discrimination at each level of warmth tested. Although the area actually varied by a factor of 10.3 , the estimated area varied by a factor between $1.63(\psi=8)$ and $2.53(\psi=20)$. This outcome illustrates the relative inferiority of the warmth sense for spatial assessment; furthermore, the lower the level of warmth, the poorer the assessment would seem to be.

A few of the Ss occasionally experienced a curious constriction of the apparent area during the course of a 3-sec exposure. The smaller the area and the higher the radiant intensity, the more likely this constriction was to be reported. (The averages in Table 1 are based on the maximal extent experienced; they are only slightly altered by using the minimal estimates instead.) When constriction occurred, it was also typically accompanied by a change in the quality of the experience. At first the warmth seemed diffuse and dull, later more localized and sharp.

Qualitative variation among the stimuli was reported by most, but not all, of the Ss. The higher levels of radiant intensity were often described as sharp or pricking (but not painful), the low levels as dull, diffuse, and pleasant. These qualitative differences probably correspond to the distinction that has frequently been made between "warmth" and "heat" (Herget \& Hardy, 1942).

\section{DISCUSSION}

The Size of the Exponent for the Warmth Sense

It is now abundantly clear that the warmth continuum cannot be characterized by a single exponent. To a first approximation, the psychophysical power rule seems to hold for any fixed area of stimulation, but the size of the exponent depends on (1) the area and (2) the body locus of stimulation. The change in the exponent as a function of area seems to behave in much the same way for forehead and back (Fig. 4). How the size of the exponent for any given area varies from one body locus to another seems to relate to local sensitivity: the better the sensitivity, the smaller the exponent. Much remains to be learned, however, about the exact relationship between local sensitivity and the exponent of the power function.

Nevertheless, the finding that locus and area influence the size of the exponent helps to reconcile what looked like conflicting results of attempts to scale warmth. The first exponents (see J.C. Stevens \& Stevens, 1963) were obtained by touching metal stimulators $\left(3.14 \mathrm{~cm}^{2}\right)$ to the forearm. In three different experiments, the exponents were 1.6, 1.8, and 1.63. Stimulation with cold yielded

Table 1

Magnitude Estimation of the Areal Extent of Warmth Stimulation

\begin{tabular}{rcccc}
\hline $\begin{array}{c}\text { Milli- } \\
\text { watt } / \mathrm{cm}^{2}\end{array}$ & 31.4 & 127 & 324 & \\
\hline \cline { 2 - 4 } 150 & 10.8 & & & \\
68 & & 15.2 & & $\psi=8$ \\
41 & & & 17.6 & \\
195 & 11.7 & & & \\
100 & & 17.2 & & $\psi=12$ \\
58 & & & 20.6 & \\
275 & 10.0 & & & \\
162 & & 18.8 & & $\psi=20$ \\
111 & & & 25.3 & \\
\hline
\end{tabular}


corresponding exponents of 1.0,1.1, and 1.02. Banks (1969) also used contact stimulation, but the area of stimulation was $19.5 \mathrm{~cm}^{2}$. This condition yielded exponents of 1.0 for warm and 0.6 for cold. Thus, the ratio of the warmth exponent to the cold exponent agreed with that found by Stevens and Stevens. The apparent disagreement on absolute size is dispelled by Fig. 4 , which shows that the smaller area $\left(3.14 \mathrm{~cm}^{2}\right)$ would be expected to yield an exponent some 1.7 times that of the larger area $\left(19.5 \mathrm{~cm}^{2}\right)$. Comparison of these two studies suggests that the exponent for cold probably also depends on area.

Other exponents have been reported for warmth: 0.7 for radiant stimulation of about $900 \mathrm{~cm}^{2}$ of the back (J.C. Stevens \& Marks, 1967) and 0.86 for radiant stimulation of the whole anterior body surface (Marks \& Stevens, 1968b). It therefore seemed that the size of the exponent might depend on whether the skin was heated by conduction or by radiation, but it now seems that the crucial factor is area. Moreover, Kenshalo, Decker, and Hamilton (1967) obtained the same degree of threshold summation under conduction and radiation. There seems little reason to retain the theory (Jenkins, 1951) that radiation and conduction act upon different receptor mechanisms or in some way trigger different modes of reaction from the same receptor mechanism.

Various attempts to scale warmth are, therefore, in first-order agreement with each other. There remains, however, the problem that all of these studies used psychophysical methods (usually magnitude estimation) that can be counted on, because of "regression" bias, to underestimate the size of the exponent The method of magnitude production would yield a larger exponent. The two methods have occasionally been paired in the effort to neutralize "regression" bias (see S.S. Stevens \& Greenbaum, 1966). Unfortunately, this has not proved possible for the thermal senses because of formidable technical difficulties associated with placing the stimulus under the direct continuous control of the $S$. To pinpoint the absolute size of the warmth exponents will, therefore, need more work Fortunately, "regression" bias does not alter the basic character of the equal-warmth functions depicted in Fig. 6 , even though these functions were generated by horizontal cuts in Figs. 2 and 5.

Is the Exponent Related to Neural Firing Rate?

Figure 4 shows, in semilog coordinates, how the obtained exponents vary with area; it is now instructive to depict the same functions in arithmetic coordinates (Fig. 11). As area becomes large, the exponent seems slowly to approach a minimal size in the vicinity of 0.7 . As area becomes small, however, the size of the exponent accelerates. The question arises whether one can estimate, by way of extrapolation, the exponent that would characterize a single receptive field.

In Fig. 11 the curves have been drawn under the assumption that the limiting exponent lies in the neighborhood of 2.0 and that the receptive fields are approximately $0.5 \mathrm{~cm}^{2}$ for the forehead and $2.0 \mathrm{~cm}^{2}$ for the back. These estimates of the receptive field sizes could fall wide of the mark without appreciably altering the prediction that the limiting exponent is approximately $2.0-$ or perhaps even larger than 2.0 .

The speculation that a single receptive field is characterized by approximately a square law is roughly consistent with what is known about the neural response rate of warm fibers serving the nasal region of the cat. Hensel and Kenshalo (1969) showed that these fibers give a highly positively accelerated response over the range from about 30 to $46 \mathrm{C}$. (With further increase above $46 \mathrm{C}$, the approximate pain threshold, the firing rate drops precipitously.) The receptive fields of this very sensitive body area measured $1.2 \mathrm{~mm}$ in diam.

\section{Two Possible Mechanisms for Summation}

The progressive loss of spatial summation with increasing sensation level may be explainable in terms of lateral neural inhibition. That spatial summation and lateral inhibition are intimately related properties is suggested by Békésy's (1967) study using two-point mechanical stimulation of the skin. According to Békisy, weak two-point stimulation is characterized by the dominance of summation, strong stimulation by lateral inhibition. In vision and hearing, the degree of lateral inhibition has been shown to increase regularly with the level of the inhibiting stimulus (S. S. Stevens, 1966). The progressive diminution of summation may, theretore, stem from the progressive augmentation of inhibition. Although little is known about inhibition in the thermal senses, Békésy (1962) offered psychophysical evidence that it does take place in the warmth sense.

An alternative explanation invokes the idea of two neural mechanisms, one that dominates at low stimulus levels and displays rich summation and another that dominates at high stimulus levels and displays little or no summation. An attractive feature of a theory of this type is that it could help to explain the qualitative variation that takes place with level of stimulation. This variation has led some investigators to postulate that the experience of "heat" is mediated by the simultaneous firing of "warmth" receptors and some other type, such as "cold" receptors or $\mathrm{c}$ fibers.

\section{Biological Function of Summation}

Some degree of spatial summation is known to take place in several sense modalities, including vision (Piéron, 1952), taste (McBurney, 1969), smell (Elsberg, 1936), cold (Hardy \& Oppel, 1938), touch (Franzén, 1969), and, of course, warmth. One biological function of summation is to permit response to weak levels of energy. In the periphery of the retina this is most likely related to the abundant convergence of the neural fibers; the excitation thresholds of the rods and the cones do not appear to differ much, because tiny areas $(2.7 \mathrm{~min})$ of the fovea and the periphery yield the same sensation threshold (Arden \& Weale, 1954). Not that peripheral convergence need be the only basis for summation; in the warmth sense a central component is needed to explain, for example, the warmth summation between the two hands (Hardy \& Oppel, 1937).

Generous summation serves to improve absolute sensitivity, but only at the sacrifice of keen spatial acuity and precise localization. One example is the poor performance of Ss called upon to estimate the area of stimulation. Another example is that the "two-point" limen for radiant stimulation of the back is extremely large, in fact, virtually indeterminate. It seems possible, however, that the ability to appreciate the spatial aspects of the stinulation may improve with increasing stimulus level.

From the point of view of body thermoregulation, however, the high sensitivity imparted by generous summation would seen to be a more advantageous property than fine acuity. Early reaction to low-level radiation distributed over large portions of the skin is important to set in motion the behavioral and physiological adjustments that serve to prevent stressful fluctuation of deep body temperatures. Within linits, it is the total heat load on the organism that matters most, not its exact distribution. Relatively high intensity confined to a small area and relatively low intensity spread out over a large area may, therefore, place essentially equivalent thermal stress on the organism (or, in the casc of a cold environment, relieve cold stress by the same amount). Accordingly, a person can feel thermally comfortable 
despite an uneven distribution of the effective environmental temperature. Portions of the body surface may gain heat, others lose heat, while overall thermal neutrality and comfort are maintained. Within certain limits, the important factor is the net thermal exchange with the environment (B $\phi j e$, Nielson, \& Olesen, 1948; Hall \& Klemm, 1969).

It is interesting that the latency of reaction to radiant stimulation depends upon level and area in much the same way as does apparent warmth. In a recent study, Banks ${ }^{2}$ showed that the simple reaction time to infrared stimulation of the forehead declines systematically with both area and intensity. That area and intensity can be traded for each other to preserve the same response latency demonstrates that summation can play a functional role in behavior without necessarily being mediated by conscious appraisal of warmth

Summation at levels well above threshold may, under some circumstances, serve to warn the organism against dangerous sources of heat and thereby serve an orienting function in behavior. By means of the galvanic skin response, Hardy, Wolff, and Goodell (1943) measured the "alarm threshold" to radiant stimulation. Although threshold varied widely from person to person, for a given person, the larger the area, the lower the alarm threshold. The same authors explain why summation would be an unfavorable property in the pain modality. Pain, even the mildest level, signifies the imminence, if not the actual incurrence, of local damage to the skin. It is essential for the organism to react quickly to the noxious stimulation, regardless of the body locus or extent involved. The absence of summation shields the organism from being so overwhelmed by massive pain as to render him incapable of proper action. Given spatial summation, minor irritation of large portions of the body surface could cause devastating pain, far out of proportion to the threat involved.

\section{REFERENCES}

ADAIR, E. R., STEVENS, J. C., \& MARKS, L. E. Thermally induced pain, the dol scale, and the psychophysical power law. American Journal of Psychology, 1968, 81, 147-164.

ARDEN, G. B., \& WEALE, R. A. Nervous mechanisms and dark-adaptation. Journal of Physiology, 1954, 125, 417-426.

BANKS, W. P. Temperature sensitivity: One subjective continuum or two? Perception \& Psychophysics, 1969, 6, 189-192.

BÉKÉSY, G. von. Lateral inhibition of heat sensations on the skin. Journal of Applied Physiology, 1962, 17, 1003-1008.

BÉKESY, G. Von. Sensory inhibition. Princeton: Princeton University Press, 1967. Chap. 2.

В $\emptyset$ JE, O., NIELSEN, M., \& OLESEN, J. Studies of the effect of unilateral cooling by radiation. Boligopvarmningsudvalgets Meddelelse Nr. 9 (Contribution No. 9 from the Committee for the Study of Domestic Heating, Copenhagen), $1948,28-30$.

ELSBERG, C. A. Monorhinal, birhinal, and bisynchronorhinal smell: The summation of impulses in birhinal smell. Bulletin of the Neurological Institute of New York, 1936, 4, 496-500.

FRANZÉN, O. On spatial summation in the tactual sense. Scandinavian Journal of Psychology, 1969, 10, 193-208.

GREENE, L. C., \& HARDY, J. D. Spatial summation of pain. Journal of Applied Physiology, 1958, 13, 457-464.

HALL, J. F., \& KLEMM, F. K. Thermal comfort in disparate thermal environments. Journal of Applied Physiology, 1969, 27, 601-606.

HARDY, J. D., \& OPPEL, T. W. Studies in temperature sensation. III. The sensitivity of the body to heat and the spatial summation of the end organ responses. Journal of Clinical Investigation, 1937, 16, 533-540.

HARDY, J. D., \& OPPEL, T. W. Studies in temperature sensation. IV. The stimulation of cold sensation by radiation. Journal of Clinical Investigation, 1938, 17, 771-778.

HARDY, J. D., WOLFF, H. G.. \& GOODELL, H. Studies on pain. A new method for measuring pain threshold: Observations on spatial summation of pain. Journal of Clinical Investigation, 1940, 19, 649-657.

HARDY, J. D., WOLFF, H. G. \& GOODELL, H. The pain threshold in man. Research Publications of the Association for Research in Nervous \& Mental Disease, 1943, 23, 1-15.

HARDY, J. D., WOLFF, H. G., \& GOODELL, H. Pain sensations and reactions. Baltimure: Williams \& Wilkins, 1952. Pp. 73-79.

HENSEL, H., \& KENSHALO, D. R. Warm receptors in the nasal region of cats. Journal of Physiology, 1969, 204, 99-112.

HERGET, C. M., GRANATH, L. P., \& HARDY, J. D. Warmth sense in relation to the area of skin stimulated. Amcrican Journal of Physiology, 1941, 135, 20-26.

HERGET, C. M., \& HARDY, J. D. Temperature sensation: The spatial sumination of heat. American Journal of Physiology, 1942, 135, 426-429.
JENKINS, W. L. Somesthesis. In S. S. Stevens (Ed.), Handbook of experimental psychology. New York: Wiley, 1951. Pp. 1172-1190.

KENSHALO, D. R., DECKER, T., \& HAMILTON, A. Spatial summation on the forehead, forearm, and back produced by radiant and conducted heat. Journal of Comparative \& Physiological Psychology, 1967, 63, 510-515.

MARKS, L. E. Brightness as a function of retinal locus in the light-adapted eye. Vision Research, 1968, 8, 525-535.

MARKS, L. E., \& STEVENS, J. C. The form of the psychophysical function near threshold. Perception \& Psychophysics, 1968a, 4, 315-318.

MARKS, L. E., \& STEVENS, J. C. Perceived warmth and skin temperature as functions of the duration and level of thermal irradiation. Perception \& Psychophysics, 1968b, 4, 220-228.

MCBURNEY. D. H. A note on the relation between area and intensity in taste. Perception \& Psychophysics, 1969, 6, 250.

MURGATROYD, D. Spatial summation of pain for large body areas. Defense Atomic Support Agency Report, October 1964.

PIÉRON, H. The sensations: Their functions, processes, and mechanisms. New Haven: Yale University Press, 1952.

STEVENS, J. C., \& MARKS, L. E. Apparent warmth as a function of thermal irradiation. Perception \& Psychophysics, 1967, 2, 613-619.

STEVENS, J. C., \& RUBIN, L. L. Psychophysical scales of apparent heaviness and the size-weight illusion. Perception \& Psychophysics, 1970, 8, 225-230.

STEVENS, J. C., \& STEVENS, S. S. Dynamics of subjective warmth and cold. In Temperature: Its measurement and control in science and industry. Vol. III, Part 3. New York: Reinhold, 1963. Pp. 239-243.

STEVENS, S. S. To honor Fechner and repeal his law. Science, 1961, 133, 80-86.

STEVENS, S. S. Power-group transformations under glare, masking, and recruitment. Journal of the Acoustical Socicty of America, 1966, 39, 725-735.

STEVENS, S. S., \& GREENBAUM, H. B. Regression effect in psychophysical judgment. Perception \& Psychoplysics, 1966, 1, $439-446$.

\section{NOTES}

1. William S. Cain, personal communication (manuscript in preparation).

2. William P. Banks, personal communication (manuscript in preparation).

(Accepted for publication Octoher 18, 1970.) 